\title{
IMPACT OF CRISIS COMMUNICATION STRATEGIES ON PEOPLE'S ATTITUDES TOWARD BEHAVIORAL GUIDELINES REGARDING COVID-19 AND ON THEIR TRUST IN LOCAL OFFICIALS
}

\author{
A Preprint
}

\author{
Gerrit Hirschfeld \\ CareTech OWL - Center for Health, Welfare and Technology, Faculty of Business \\ University of Applied Sciences Bielefeld \\ Interaktion 1, 33619 Bielefeld, Germany \\ gerrit.hirschfeld@fh-bielefeld.de \\ Meinald T. Thielsch \\ Department for Psychology \\ University of Münster \\ Fliednerstr. 21, 48149 Münster, Germany \\ thielsch@wwu.de
}

June 1, 2021

\begin{abstract}
Introduction: The communication patterns of commercial organizations are generally guided by Situational Crisis Communication Theory (SCCT), but the impact of different crisis communication strategies for public messaging on COVID-19 has not been thoroughly examined. As such, we test how crisis communication strategies affect trust in mayors and the acceptance of behavioral measures, specifically regarding the buffering effect of a mayor's pre-crisis reputation as well whether trust mediates the link between crisis communication strategies and acceptance of behavioral measures.

Methods: A total of 561 participants (53\% female; mean age 50 yrs) took part in an online experiment in which we systematically manipulated the mayor's crisis communication strategy (deny vs. diminish, vs. rebuild, vs. bolstering, vs. no response) and pre-crisis reputation (good past crisis management, bad past crisis management). Age, gender and education served as covariates. In an exploratory analysis, we also tested the predictive power of personal concern regarding the COVID-19 pandemic as well as internal and external control convictions.

Results: In our pre-planned analysis, we found that crisis communication strategies and pre-crisis reputation had no significant effect on participants' ratings of acceptance of certain behaviors or their behavioral intentions. However, the different communication strategies did affect participants' trust in the mayor and intention to vote for him. Specifically, we found that while the strategy of denying was overall unsuccessful, all other strategies fared similarly when the mayor's pre-crisis reputation was high. When his pre-crisis reputation was low, differences emerged between the other strategies. The exploratory analysis corroborated earlier findings about the importance of individual concern and trust as predictors of behavioral measures. Discussion: Overall, SCCT seems to be an adequate description the effects of communication strategies on reputation in local officials during a pandemic. Yet, neither direct effects of communication strategies on acceptance of behavioral measures nor indirect effects on behavioral measures could not be shown. Since trust is an important aspect, we advise local officials to carefully choose their communication style.
\end{abstract}


A PREPRINT - JunE 1, 2021

Keywords Crisis Communication · Situational Crisis Communication Model · COVID-19 


\section{Introduction}

When effective and widely available vaccines are lacking, the spread of a pandemic is best mitigated through behavioral measures such as keeping a distance from and limiting one's contact with other people (Ferguson et al., 2020). Accordingly, several studies have tried to identify demographic variables that are associated with people's acceptance and adoption of key behavioral measures, such as keeping a distance, wearing face masks, regularly washing hands, etc. (Dohle et al., 2020; van Mulukom, n.d.). However, while such knowledge is important from a theoretical perspective, it cannot easily be translated into actual actions that political or private actors should take, mainly for two reasons. First, existing studies have focused on variables such as party preference, religiosity, or trust, and these cannot easily be changed. Second, these studies have mainly used correlational designs, which do not test the impact of specific interventions. As far as we know, only two previous studies have investigated the impact of different forms of messaging on people's intentions to engage in various behavioral measures to prevent COVID-19 (Capraro and Barcelo, 2020; Everett et al., 2020). These studies manipulated the reasons that were given for why people should adhere to behavioral measures, but they were not able to show clear effects of different messages on people's intention to adhere to several behavioral measures - washing hands, keeping a distance, etc. In the present study, we use a similar experimental paradigm to test the impact of different crisis communication strategies on participants' acceptance of and intention to engage in different behavioral measures.

Crisis communication is a well-established research domain that studies how the public perception of incidents can be shaped. The most influential theory of crisis communication is the Situational Crisis Communication Theory (SCCT; for a review see: Coombs, 2006). SCCT was developed in the 1990s by Coombs and Holladay (Coombs and Holladay, 1996) to address questions of corporate responsibility in the face of crises, e.g., reports of damaged products or mistreatment of employees, etc. The basic tenant of SCCT is that the communication style of an organization has to match the specific type of crisis that the organization faces. To this end, SCCT defines different clusters of crises that mostly differ in the perceived control an organization has over the crisis. Depending on the crisis cluster, different response strategies can be used (Coombs, 2006), the most important of which are (1) denying a crisis exists, (2) diminishing one's own role in the crisis, (3) rebuilding the relationship after taking responsibility for the crisis (4) bolstering one's reputation by reminding others of past positive behavior and (5) giving no response. A large body of empirical research has investigated the direct effect of crisis communication strategies and possible moderating variables (e.g., Beldad et al., 2018; Coombs, 2006; Coombs and Holladay, 1996; Hegner et al., 2016). Research has revealed that a positive pre-crisis reputation can buffer the effects of a crisis and of misaligned crisis communication strategies (Hegner et al., 2014). Even though this theory has been developed in the corporate domain, it can also be used to study how public actors respond in times of crisis: In fact, Coombs himself recently highlighted the specific crisis communication demands of COVID-19 (Coombs, 2020). We aim to use SCCT to analyze how communication styles affect the reputations of local officials, such as mayors - in which a central aspect of these reputations is trust - and affect people's acceptance of and intention to engage in behavioral measures (Gefen et al., 2008; Mayer et al., 1995).

Trust is indispensable for effective communication between political leaders and their subjects (Lewis and Weigert, 1985). Further, a previous review into different predictors of behavioral measures found that in the context of a pandemic, trust is an important predictor of behaviors (Bish and Michie, 2010). Likewise, several recent studies have highlighted the important role of trust in people's acceptance of behavioral measures against COVID-19 (Christensen and Lægreid, 2020; Dohle et al., 2020; Guglielmi et al., 2020; van Mulukom, n.d.). While some of these studies emphasize complex interactions between trust in politicians and acceptance of behavioral measures, i.e., the paradox of confidence (Guglielmi et al., 2020), some studies also support the idea that the public's acceptance of behavioral measures is positively related to trust in politicians and scientists (Dohle et al., 2020; Guglielmi et al., 2020; van Mulukom, n.d.). Further, it can be argued that trust and trustworthiness are distinct, as they can vary independently (Gefen et al., 2008; Mayer et al., 1995; Meeßen et al., 2021): For instance, if a person has a generally low disposition toward trusting politicians, they might even distrust a political leader that is highly trustworthy.

The aim of the present study is to test whether crisis communication strategies can be used to influence mayors' reputations and the public's acceptance of and intention to engage in behavioral measures. We chose mayors because, as local officials, they are responsible for implementing governmental policies to protect the public and for overseeing the implementation of local decrees (such as mask mandates or restricting public events). Further, mayors have often led the local crisis management teams responsible for managing the pandemic response (Thielsch et al., 2021). As demonstrated by the actual responses of mayors in Italy ("'This is not a film,", 2020) and Germany ("Corona," 2020), large differences exist in the crisis communication 
styles employed. Our hypotheses are based on SCCT (Coombs, 2006) as well as past experimental research into how different forms of leaders' messaging affect the public: Based on (Capraro and Barcelo, 2020; Everett et al., 2020), we assume that different crisis communication styles produce differences in people's behavioral intentions to engage in (H1a) and accept (H1b) protective behavioral measures, in how they rate the trustworthiness of the mayor (H1c: benevolence; H1d: integrity; H1e: competence), in their trust in the mayor (H1f) and in their intention to vote (H1g). Our second hypotheses (H2a-g) are similarly based in SCCT, specifically in research showing that a leader's pre-crisis reputation can have a buffering effect (Beldad et al., 2018; Hegner et al., 2016), and they state that the above differences (H1a-g) are moderated by the mayor's pre-crisis reputation, where a high pre-crisis reputation buffers the effects of the crisis communication; i.e., we expect larger differences in H1a-g when the mayor's pre-crisis reputation is low. Our third set of hypotheses is further based on studies showing strong associations between trust and adherence to behavioral measures (Dohle et al., 2020; Guglielmi et al., 2020; van Mulukom, n.d.); they state that differences in acceptance (H3a) and behavioral intentions ( $\mathrm{H} 3 \mathrm{~b})$ are mediated by overall trust. In addition, we ran two exploratory analyses. The first aimed to test whether weaknesses in our experimental manipulation contributed to the lack of significant effects. The second aimed to investigate whether demographic variables, control beliefs and personal concerns related to COVID-19 influenced people's behavioral intentions.

\section{Methods}

\subsection{Participants}

Participants were recruited via the German online panel PsyWeb (https://psyweb.uni-muenster.de/). Participating in this panel is completely voluntarily, and members agree to receive invitations for scientific studies; they can unsubscribe and delete their personal data at any time. Further, as no major changes were made between the pre-test questionnaire and the actual study questionnaire, the eight respondents who took the pre-test questionnaire were included in the study. Of 886 participants who started the questionnaire, 623 completed the full survey. Next, 62 participants were excluded from further analysis due to unrealistically short response durations, no variance in their responses, or a wrong answer to an attention-check item (Meade and Craig, 2012). The final dataset, which included 561 participants, is available for download at zenodo https://doi.org/10.5281/zenodo.4889821

\subsection{Manipulating the crisis communication strategy and the mayor's pre-crisis reputation}

Participants read one out of a total of ten different versions of a newspaper article describing a localized surge in COVID-19 cases. The different versions of the newspaper article were modeled after one article describing an outbreak in the German city of Hamm in October 2020. All articles started with a short introductory paragraph describing a rapid increase in cases in the fictional town of Sonnenfels (the article mentioned 650 cases/wk/100,000 inhabitants; the German average infection rate at the time of the study was around 300 cases/wk/100,000 inhabitants). After this, the article described three novel behavioral measures that were introduced in the city: People in the town were expected to (1) wear masks in public at all times, (2) register private gatherings containing more than 10 people as well as cancel/not have private parties with more than 20 people and (3) not attend Sonnenfels's annual village fair and the open Sunday, as the city canceled these events. (These behavioral measures were much stricter than restrictions that were actually in place in Germany at the time of data collection.) After these sections of the article, the two-part manipulation began. Namely, we manipulated how the article described the mayor's specific communication strategy (deny, diminish, rebuild, bolster, no response) and the mayor's pre-crisis reputation (high vs. low); this resulted in ten different versions of the article. Participants were randomly allocated to the conditions, resulting in sample sizes for each condition between 52 and 61 (median $=56$ ) participants. The study was available online from 6 until 30 November 2020, with most subjects $(88.04 \%)$ participating in the first week of the field period. Completing the study on average took about seven to nine minutes (median $=7.46 \mathrm{~min} ; \mathrm{M}=$ $8.77 \min , \mathrm{SD}=6.18)$.

\subsection{Measures}

After participants read the description, they were asked to respond to a series of questionnaires. For a complete list of items and instructions see: https://doi.org/10.5281/zenodo.4889821 
Table 1: Overview of Manipulation

\begin{tabular}{|c|c|c|}
\hline Condition & description & Manipulation \\
\hline Denial & $\begin{array}{l}\text { Claim that no crisis } \\
\text { exists }\end{array}$ & $\begin{array}{l}\text { In my opinion the numbers cannot be used to } \\
\text { draw strong conclusions about the risks of the } \\
\text { public in Sonnenfels. First, mostly young } \\
\text { persons are infected for whom COVID is not } \\
\text { dangerous. Second, the rise in numbers can be } \\
\text { attributed to increased testing. }\end{array}$ \\
\hline Diminish & $\begin{array}{l}\text { Playing down the } \\
\text { cities responsibility }\end{array}$ & $\begin{array}{l}\text { We as a city do only have a limited impact on } \\
\text { the infections. Many, e.g. those commuting to } \\
\text { work in other cities, get infected there but are } \\
\text { counted as cases here. It is also self-evident } \\
\text { that if the numbers go up in neighboring cities, } \\
\text { that numbers in Sonnenfels will go up, too. }\end{array}$ \\
\hline Rebuild & $\begin{array}{l}\text { Taking } \\
\text { responsibility }\end{array}$ & $\begin{array}{l}\text { We as city are well aware of our responsibility } \\
\text { towards the inhabitants and we will do our } \\
\text { best to match the increase in the. We } \\
\text { requested help from other cities, increased our } \\
\text { testing capacities and optimized our internal } \\
\text { organisation. }\end{array}$ \\
\hline Bolstering & $\begin{array}{l}\text { Remind public of } \\
\text { past good }\end{array}$ & $\begin{array}{l}\text { Sonnenfels is a city that cares for it's } \\
\text { inhabitants. During the first wave of COVID } \\
\text { we did a very good job and given the tireless } \\
\text { efforts by our city clerks, we have avoided the } \\
\text { worst of this crisis. However, also we as city } \\
\text { are not able to work at full capacity due to } \\
\text { many ill colleagues. }\end{array}$ \\
\hline $\begin{array}{l}\text { No re- } \\
\text { sponse }\end{array}$ & No response & $\begin{array}{l}\text { The new rules were published on the cities web } \\
\text { site. The major was not availible for further } \\
\text { comments. }\end{array}$ \\
\hline
\end{tabular}

\subsubsection{Trustworthiness of the mayor}

To measure the trustworthiness of the mayor, we used the TrustDiff scale (Brühlmann, 2019). The TrustDiff is a semantic differential consisting of ten items that assess three different dimensions of trust: benevolence (e.g.,ignoring - caring"), integrity (e.g., "dishonest - honest") and competence (e.g., "inept - resourceful"). Participants used a seven-point scale to indicate for each of the ten word pairs which of the two descriptors was more fitting for the mayor. The TrustDiff has been extensively validated to measure user trust in web settings and has good psychometric properties. Cronbach's alpha in the present sample was .89, .93, and .93 for the benevolence, integrity, and competence scales, respectively.

\subsubsection{Trust in the mayor}

To measure trust in the mayor, we posed three direct questions regarding the mayor ("I can trust the mayor." "I rely on what the mayor says." "I have no reservation about relying on what the mayor says."). Cronbach's alpha in the sample was .95 . Furthermore, we asked participants to indicate on a scale from $0 \%$ to $100 \%$ how likely they would be to vote for this mayor in an upcoming election.

\subsubsection{Adherence and intention measures}

The adherence and intention measures were based on the questionnaire developed by Dohle and colleagues (2020). Both questionnaires consist of nine items naming different behavioral measures (keeping a distance from other persons, regularly washing hands with soap, sneezing into the arm, wearing a mask in public, working from home, canceling private meetings with more than 20 persons, registering personal meetings with more than 10 persons, avoiding large gatherings, avoiding contact with other people). The measures were selected because they were widely discussed as possible next steps in the COVID-19 response at the time of the study. For the adherence measure, participants were asked to rate the utility of the different behavioral 
measures on a scale from 1 (utterly useless) to 7 (very useful). For the intention measure, participants were asked to estimate how often they would comply with these behavioral measures on a scale from 1 (never) to 5 (always). Cronbach's alpha within the present sample was .81 for the acceptance measure and .79 for the intention measure.

\subsubsection{Perceived control and level of personal concern regarding the COVID-19 pandemic}

To measure perceived control, we used the IE-4 scale that measures internal and external control convictions Kovaleva et al. (2014). The scale consists of four items that measure two scales, namely internal control convictions (e.g., "I'm my own boss.") and external control convictions (e.g., "Fate often gets in the way of my plans"). Furthermore, we used a single item "How concerned are you about the current COVID-19 situation?" to which the participants responded on a scale of 0 ("not at all concerned") to 6 ("very concerned").

\subsubsection{Attention and manipulation check}

Lastly, we used three additional items to check the manipulation and participants' attention. The manipulation check consisted of two single-choice items. The first asked participants to summarize the statement the mayor gave as part of the newspaper article. Participants could choose one of five different descriptions, each of which described one communication style. The second item asked whether the mayor's past performance was positive or negative.

Attention was checked by including one item into the ratings for the mayor that stated "Please select option 1 for this item for quality control" (Meade and Craig, 2012).

\subsection{Data-Analysis}

The data analysis was conducted in accordance with analysis plan that was established and pre-registered before data collection began

(see https://aspredicted.org/blind.php? $\mathrm{x}=\mathrm{bw6cz} 7$ ). H1a-e and H2a-e were tested using a $5^{*} 2$ MANCOVA (DVs: intention, acceptance, trust-Ability, trust-Benevolence, trust-Intention; AV1: crisis communication style; AV2: pre-crisis reputation; control variables: age, gender, education). Whenever the MANCOVA yielded significant overall results, these were followed-up on using corresponding separate ANCOVAs for the individual DVs. Relevant to the evaluation of H1a-g were the main effects of crisis communication style. Relevant to the evaluation of H2a-g were the crisis communication style by pre-crisis reputation interactions. The effect sizes are reported as omega squared. H3 was tested using two separate mediation analyses, one for acceptance and one for behavioral intentions. In both analyses, we estimated the average causal mediation effect (ACME) via the mediation package in R (Tingley et al., 2014). The mediator model described the mediator trust as a function of crisis communication style, level of control and level of anxiety about the pandemic. The outcome model described the level of acceptance (H3a) and the behavioral intentions (H3b) as a factor of the mediator trust, the crisis communication style and level of perceived control and anxiety about the pandemic. The conditions 1-4 (deny, diminish, rebuild, and bolster) were individually contrasted to condition 5 (no response). The significance of ACMEs were assessed using the bca-corrected bootstrap.

Additionally, we ran two exploratory analyses. The first used participants' perceptions of the communication strategy as assessed in the manipulation check instead of the assigned condition. The second used hierarchical regressions to predict behavioral measures using demographic characteristics (step 1), control convictions (step 2) personal concern regarding the COVID-19 pandemic (step 3) and trust (step 4).

The study was approved by the ethics board of the University of Münster's Faculty 7, Psychology \& Sports Science (2020-57-MT). All materials, raw data and analysis scripts are publicly accessible https: //doi.org/10.5281/zenodo.4889821.

\section{Results}

\subsection{Manipulation check}

Overall, the manipulation check indicated that the majority of participants chose the intended descriptor for the deny, rebuild and no-response categories, but not for the diminish and bolster categories (see tab. 2). For both these conditions, the majority of participants instead chose rebuild as the best summary. In contrast, the vast majority $(81 \%)$ stated that they perceived the manipulation about the mayor's past performance as intended in the study design. 
A PREPRINT - JunE 1, 2021

Table 2: Responses to the manipulation check item in the different conditions

\begin{tabular}{lrrrrr}
\hline man_check & 1. deny & 2. diminish & 4. bolstering & 5. no response & 3. rebuild \\
\hline Deny & 50 & 1 & 1 & 1 & NA \\
Diminish & 5 & 31 & 4 & 2 & 1 \\
Rebuild & 26 & 49 & 57 & 39 & 82 \\
Bolstering & 13 & 16 & 38 & 11 & 22 \\
No response & 10 & 5 & 5 & 58 & 11 \\
Do not know & 5 & 5 & 2 & 6 & 2 \\
\hline
\end{tabular}

\section{$3.2 \quad$ Pre-specified analysis}

Hypotheses about the main effects of communication strategy (H1) and the buffering effect of pre-crisis reputation (H2) were tested using an MANCOVA to compare the mean in the different conditions (see fig. 1). The overall MANCOVA revealed significant effects for strategy (Pillais' Trace $=.23 ; \mathrm{F}(24,2188)=5.59 ; \mathrm{p}<$ $.001 ; \omega=0.06$ ), pre-crisis reputation (Pillais' Trace $=.10 ; \mathrm{F}(6,544)=9.82 ; \mathrm{p}<.001 ; \omega=0.10$ ) and age (Pillais' Trace $=.04 ; \mathrm{F}(6,544)=3.74 ; \mathrm{p}<.01 ; \omega=0.04)$. The follow-up analyses for the acceptance (H1a, $\mathrm{H} 2 \mathrm{a})$ and intention (H1b, H2b) measures showed small but significant effects for sex and age but not for the experimental variables or interactions (all p values $>.1$ ). Women and older participants had higher ratings of acceptance and intention.

In contrast, participants' ratings of the mayor's benevolence (H1c), integrity (H1d), competence (H1e), trust (H1f) and their intention to vote was affected by the strategy manipulation. Specifically, we found significant main effects for strategy (H1c-f) and pre-crisis reputation for all variables. The strategy effect was driven by the fact that the deny strategy yielded more negative ratings than the diminish, rebuild, bolstering and no-response strategies, which did not differ much from one another. The main effect for pre-crisis reputation was due to more positive ratings for the positive reputation conditions. Furthermore, we also found the hypothesized interactions between strategy and pre-crisis reputation for the variables integrity (H2d) and trust (H2f). This interaction occurred due to the much larger differences between the diminish, rebuild, bolster and no-response strategies in the negative pre-crisis reputation (PCR) condition than in the positive PCR condition. Bolstering specifically evoked almost as negative of ratings as did deny in the negative condition, but it was perceived as much more positive than deny was in the positive condition.

Of the planned mediation analysis (H3) all but those contrasting the no-response condition to rebuild yielded insignificant estimates of the ACME (intention: est $=-0.09 ; 95 \% \mathrm{CI}=-.16$ to $-.03 ; \mathrm{p}<.001$; acceptance: est $=-0.09 ; 95 \% \mathrm{CI}=-.17$ to $-.01 ; \mathrm{p}=.02)$. Participants in the bolstering condition gave higher trust ratings than participants in the no-response condition and participants who gave higher trust ratings in turn gave higher ratings of behavioral measures. Taken together our hypothesis regarding the effects on acceptance of and adherence to behavioral measures could not be supported, while the hypotheses regarding the effect on trust in local officials were partially supported. 


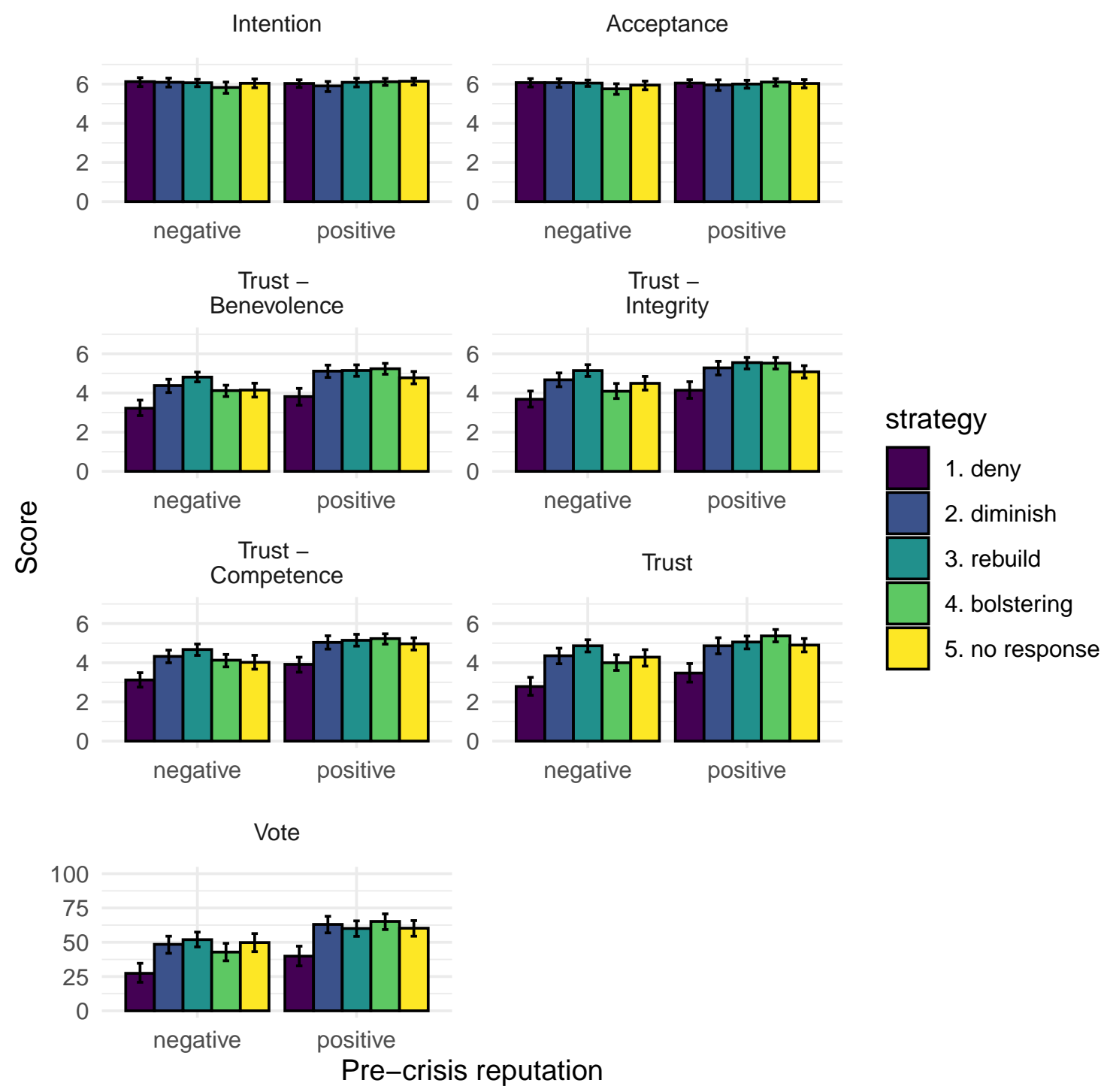

Figure 1: Mean scores for all conditions. Error bars indicate 95\%CI

\subsection{Exploratory Analysis}

Following our results, we also performed two additional analyses. First, to exclude the possibility that our manipulation was misunderstood by some participants, we used the participants' perception of the communication strategy instead of the assigned experimental condition. This, however, did not alter the pattern of results. Importantly, the behavioral ratings were not significantly different.

Second, we performed a hierarchical regression analysis to predict participants' acceptance of (tab. 3) and intention to perform (tab. 4) the behavioral measures. Both yielded similar results, in that both demographic variables, control convictions, personal concern regarding the COVID-19 pandemic and trust all contributed to the prediction of the behavioral measures. Upon inspecting the variance accounted for in these models, we found that the level of personal concern regarding the COVID-19 pandemic was the single largest contributing variable. Personal concern accounted for $20 \%$ of the variance in acceptance - and $15 \%$ in intentions - above and beyond demographic characteristics and control convictions. Notably, while the amount of incremental variance accounted for by trust in the mayor was low (only about 4\%) this amount was still significant (intention: $\mathrm{F}(1,553)=28.22 ; \mathrm{p}<.001$; acceptance: $\mathrm{F}(1,553)=15.90 ; \mathrm{p}<.001)$. 
Table 3: Results of the hierarchical regression analysis predicting acceptance of behavioral measures

\begin{tabular}{|c|c|c|c|c|}
\hline & (1) & (2) & (3) & (4) \\
\hline \multirow[t]{2}{*}{ (Intercept) } & $6.001 * * *$ & $5.692 * * *$ & $4.789 * * *$ & $4.598 * * *$ \\
\hline & $(0.243)$ & $(0.402)$ & $(0.365)$ & $(0.356)$ \\
\hline \multirow[t]{2}{*}{ sex } & $-0.252^{* * *}$ & $-0.269 * * *$ & $-0.157 *$ & $-0.177 * *$ \\
\hline & $(0.070)$ & $(0.070)$ & $(0.063)$ & $(0.061)$ \\
\hline \multirow[t]{2}{*}{ age } & 0.004 & 0.005 & 0.003 & 0.001 \\
\hline & $(0.002)$ & $(0.002)$ & $(0.002)$ & $(0.002)$ \\
\hline \multirow[t]{2}{*}{ education } & 0.038 & 0.034 & 0.021 & 0.018 \\
\hline & $(0.039)$ & $(0.039)$ & $(0.034)$ & $(0.034)$ \\
\hline \multirow[t]{2}{*}{ internal } & & $0.085 *$ & 0.055 & 0.044 \\
\hline & & $(0.042)$ & $(0.037)$ & $(0.036)$ \\
\hline \multirow[t]{2}{*}{ external } & & -0.042 & $-0.104 * * *$ & $-0.107 * * *$ \\
\hline & & $(0.034)$ & $(0.030)$ & $(0.030)$ \\
\hline \multirow[t]{2}{*}{ concern } & & & $0.320 * * *$ & $0.301^{* * *}$ \\
\hline & & & $(0.026)$ & $(0.026)$ \\
\hline \multirow[t]{2}{*}{ trust2 } & & & & $0.104 * * *$ \\
\hline & & & & $(0.018)$ \\
\hline $\mathrm{N}$ & 561 & 561 & 561 & 561 \\
\hline $\mathrm{R} 2$ & 0.025 & 0.044 & 0.248 & 0.290 \\
\hline
\end{tabular}

*** $\mathrm{p}<0.001 ;{ }^{* *} \mathrm{p}<0.01$ * $^{*} \mathrm{p}<0.05$. 
A PREPRINT - JUNE 1, 2021

Table 4: Results of the hierarchical regression analysis predicting intention to adhere to behavioral measures

\begin{tabular}{|c|c|c|c|c|}
\hline & (1) & $(2)$ & (3) & (4) \\
\hline \multirow[t]{2}{*}{ (Intercept) } & $5.832 * * *$ & $5.791 * * *$ & 4.990 *** & $4.797 * * *$ \\
\hline & $(0.251)$ & $(0.418)$ & $(0.393)$ & $(0.385)$ \\
\hline \multirow[t]{2}{*}{ sex } & $-0.271 * * *$ & $-0.280 * * *$ & $-0.180^{* *}$ & $-0.201 * *$ \\
\hline & $(0.072)$ & $(0.073)$ & $(0.067)$ & $(0.066)$ \\
\hline \multirow[t]{2}{*}{ age } & $0.005 *$ & $0.005 *$ & 0.004 & 0.002 \\
\hline & $(0.003)$ & $(0.003)$ & $(0.002)$ & $(0.002)$ \\
\hline \multirow[t]{2}{*}{ education } & 0.076 & 0.073 & 0.062 & 0.059 \\
\hline & $(0.040)$ & $(0.040)$ & $(0.037)$ & $(0.036)$ \\
\hline \multirow[t]{2}{*}{ internal } & & 0.032 & 0.005 & -0.006 \\
\hline & & $(0.044)$ & $(0.040)$ & $(0.039)$ \\
\hline \multirow[t]{2}{*}{ external } & & -0.034 & $-0.089 * *$ & $-0.092 * *$ \\
\hline & & $(0.035)$ & $(0.033)$ & $(0.032)$ \\
\hline \multirow[t]{2}{*}{ concern } & & & $0.284 * * *$ & $0.265 * * *$ \\
\hline & & & $(0.028)$ & $(0.028)$ \\
\hline \multirow[t]{2}{*}{ trust2 } & & & & $0.104^{* * *}$ \\
\hline & & & & $(0.020)$ \\
\hline $\mathrm{N}$ & 561 & 561 & 561 & 561 \\
\hline $\mathrm{R} 2$ & 0.032 & 0.038 & 0.187 & 0.227 \\
\hline
\end{tabular}

\section{Discussion}

The aim of the present study was to test the predictions of SCCT in the domain of COVID-19. Overall, our hypothesis (H1a, H1b) regarding the effect of communication strategy on behavioral measures (both acceptance and intention) have to be rejected, because the different communication strategies used by mayors did not affect participants' ratings of the behavioral measures. Similarly, we only found limited support for the buffering effect of the mayor's pre-crisis reputation (H2) and no support for the hypothesized mediation effects (H3). However, we did find the expected effects of crisis communication strategies on the evaluations of mayors (H1c-H1e). Our exploratory analysis showed that trust in the mayor was related to participants' acceptance of and intention to adhere to behavioral measures, but the single biggest predictor was the level of personal concern about the COVID-19 pandemic. In the following, we first discuss the absence of the predicted effects on the behavioral ratings, and after that we discuss the effects of communication strategy on trust and the relationship between level of concern, trust and behavioral measures. Finally, we describe some limitations of the study and provide a general outlook. 
Similar to earlier studies (Capraro and Barcelo, 2020; Everett et al., 2020), we did not find the predicted effects of message type on participants' behavioral intentions. This lack of evidence might be due to the "minimal" intervention that was used to differentiate between the crisis communication styles. In the present study, this was particularly highlighted in the manipulation check, where many participants did not accurately choose the crisis communication strategy that the condition intended to depict. Importantly, participants seemed to be biased toward the rebuild strategy, which could be because this strategy is centered around initiating real change. Since the mayors' statements were made in the context of introducing novel behavioral measures, it is understandable that participants also used this information when responding to the manipulation-check items. However, as our exploratory analysis indicates, the predicted effects were also absent when we used participants' descriptions of the communication conditions instead of the intended communication conditions. While the lack of significant effects cannot, strictly speaking, be interpreted as a lack of effects, we believe that together with similar results from other studies, the present results indicate that variations in message wordings (Capraro and Barcelo, 2020; Everett et al., 2020) alone are not enough to drive substantial effects on behavioral intentions. Nevertheless, we must still consider that our manipulation was based on actual real newspaper reports; as such, this type of informational text would be used in practice, but presumably (compared to our study) it would reach more readers.

We did, however, find that information about a mayor's pre-crisis reputation and the mayor's crisis communication style affected participants' trust in the mayor and their intention to vote for the mayor in upcoming elections: A mayor who was described as leading an effective response to COVID-19 was trusted more than a mayor whose past response was described as deficient. At the same time, communication patterns can strongly shape the perception of a mayor, such that even a mayor with a bad record on crisis management can garner some level of trust by choosing the right communication strategy. Echoing previous studies (Coombs, 2020; Coombs and Holladay, 1996), we found that the rebuild strategy was particularly effective when a mayor's pre-crisis reputation was low. Bolstering was only effective when the pre-crisis reputation was high but not when it was low. The worst strategy with regard to trustworthiness, trust and intention to vote was denying that a specific problem existed. However, we also found that in the context of a pandemic, diminishing one's responsibility by scapegoating other cities was also highly effective. Yet, while this strategy might help individual mayors in the short-term, it has harmful long-term effects on the overall societal coherence.

In line with other researchers (Dohle et al., 2020; Harper et al., 2020), we found that several demographic and attitudinal variables were related to participants' acceptance of and adherence to behavioral measures. However, the magnitude of these associations in terms of the amount of variance they explained differed. Dohle and colleagues found that perceived risk of infection only accounted for about $3 \%$ to $5 \%$ of incremental variance explained in acceptance of and adherence to behavioral measures beyond that explained by demographic characteristics. Trust in politics added another 16\% and 11\% to the variance explained in acceptance of and adherence to behavioral measures. We found a much stronger association between concern and acceptance and adherence to behavioral measures, i.e., concern explained $20 \%$ of the accounted for variance, while trust only added another 4\%. While Harper and colleagues (Harper et al., 2020) did not use trust as a predictor of adherence to behavioral measures, their reported correlation between fear of COVID-19 and adherence was similar to the association reported here between adherence and concern. To harmonize these disparate findings, it will be important to develop a shared set of measures.

The present study also highlights some specific problems for research that examines participants' acceptance of and adherence to behavioral measures. First, all studies (Capraro and Barcelo, 2020; Dohle et al., 2020; Everett et al., 2020) have reported ceiling effects of the behavioral measures, because a vast majority of participants reports high levels of acceptance. Given the general importance of the COVID-19 pandemic and the fact that some of these behaviors are being criminalized, it may be necessary to use techniques that minimize the effects of social desirability, e.g., randomized response techniques (Fox and Tracy, 1986). Second, studies need to take into account the source of the message. While behavioral measures have to be implemented and enforced at a local level, national government bodies are, of course, also an important source of information for citizens. In Germany - as in many other countries - the relationship between the different levels of government has shifted over time; at the beginning of the pandemic, there was some tension between the different local and national levels of government (Thielsch et al., 2021). Apparently, countries that managed the COVID-19 pandemic relatively successfully were able to account for the actual capacity for action at the different levels (Christensen and Lægreid, 2020).

\subsection{Conclusions}

The COVID-19 pandemic has placed a heavy burden not only on the health care system but on the public sector at large. In this context, leaders' success in implementing behavioral measures relies on trust and 
effective transparent communication strategies (Christensen and Lægreid, 2020; Thielsch et al., 2021). The present study adds to the emerging field of studies regarding which messages are most effective (Capraro and Barcelo, 2020; Everett et al., 2020). While we found that isolated statements alone do have measurable effects on people's trust in politicians, they only minimally affect people's acceptance of and adherence to behavioral measures.

\section{Declarations}

\subsection{Acknowledgements}

We would like to thank Sabrina Hegner for helpful discussions in preparing this work, Christoph Lamers for feedback on the validity of the different mayoral responses, Johanna Bunk for her support in preparing the online supplements and Celeste Brennecka for proofreading the manuscript.

\subsection{Conflicts of Interest}

The authors declare no conflicts of interest.

\subsection{Availability of data, code and materials}

The raw data, R-codes to replicate the analysis and the questionnaire is available at: https://doi.org/10 $5281 /$ zenodo.4889821

\subsection{Pre-registration}

The complete pre-registration is accessible at: https://aspredicted.org/blind.php? $\mathrm{x}=\mathrm{bw6}$ cz7 . Hypotheses H1f and H2f and H1g and H2g were added after the pre-registration (but before starting data collection) to better differentiate between trustworthiness and trust.

\section{References}

Beldad, A.D., van Laar, E., Hegner, S.M., 2018. Should the shady steal thunder? The effects of crisis communication timing, pre-crisis reputation valence, and crisis type on post-crisis organizational trust and purchase intention. Journal of contingencies and crisis management 26, 150163.

Bish, A., Michie, S., 2010. Demographic and attitudinal determinants of protective behaviours during a pandemic: A review. British journal of health psychology 15, 797824.

Brühlmann, F., 2019. Understanding and improving subjective measures in human-computer interaction ( $\mathrm{PhD}$ thesis).

Capraro, V., Barcelo, H., 2020. The effect of messaging and gender on intentions to wear a face covering to slow down COVID-19 transmission. arXiv preprint arXiv:2005.05467.

Christensen, T., Lægreid, P., 2020. Balancing governance capacity and legitimacy: How the norwegian government handled the COVID-19 crisis as a high performer. Public Administration Review 80, 774779.

Coombs, W.T., 2006. Crisis management: A communicative. Public relations theory II 149.

Coombs, W.T., 2020. Public sector crises: Realizations from covid-19 for crisis communication. Partecipazione e conflitto 13, 9901001.

Coombs, W.T., Holladay, S.J., 1996. Communication and attributions in a crisis: An experimental study in crisis communication. Journal of public relations research 8, 279295.

Corona: Bürgermeister fordert Bürger zum Daheimbleiben auf, 2020. Westdeutschel Allgemeine Zeitung.

Dohle, S., Wingen, T., Schreiber, M., 2020. Acceptance and adoption of protective measures during the covid-19 pandemic: The role of trust in politics and trust in science.

Everett, J.A., Colombatto, C., Chituc, V., Brady, W.J., Crockett, M., 2020. The effectiveness of moral messages on public health behavioral intentions during the COVID-19 pandemic. 
Ferguson, N., Laydon, D., Nedjati Gilani, G., Imai, N., Ainslie, K., Baguelin, M., Bhatia, S., Boonyasiri, A., Cucunuba Perez, Z., Cuomo-Dannenburg, G., 2020. Report 9: Impact of non-pharmaceutical interventions (NPIs) to reduce COVID19 mortality and healthcare demand.

Fox, J.A., Tracy, P.E., 1986. Randomized response. A method for sensitive surveys. Beverly Hills: Sage, Quantitative Applications in the Social Sciences.

Gefen, D., Benbasat, I., Pavlou, P., 2008. A research agenda for trust in online environments. Journal of Management Information Systems 24, 275-286.

Guglielmi, S., Dotti Sani, G.M., Molteni, F., Biolcati, F., Chiesi, A.M., Ladini, R., Maraffi, M., Pedrazzani, A., Vezzoni, C., 2020. Public acceptability of containment measures during the COVID-19 pandemic in italy: How institutional confidence and specific political support matter. International Journal of Sociology and Social Policy 40, 1069-1085. doi:10.1108/IJSSP-07-2020-0342

Harper, C.A., Satchell, L.P., Fido, D., Latzman, R.D., 2020. Functional fear predicts public health compliance in the COVID-19 pandemic. International journal of mental health and addiction 114.

Hegner, S.M., Beldad, A.D., Kraesgenberg, A.-L., 2016. The impact of crisis response strategy, crisis type, and corporate social responsibility on post-crisis consumer trust and purchase intention. Corporate Reputation Review 19, 357370.

Hegner, S.M., Beldad, A.D., op Heghuis, S.K., 2014. How company responses and trusting relationships protect brand equity in times of crises. Journal of brand management 21, 429445.

Kovaleva, A., Beierlein, C., Kemper, C.J., Rammstedt, B., 2014. Internale-externale-kontrollüberzeugung-4 (IE-4).

Lewis, J.D., Weigert, A., 1985. Trust as a social reality. Social forces 63, 967985.

Mayer, R.C., Davis, J.H., Schoorman, F.D., 1995. An integrative model of organizational trust. Academy of Management Review 23, 709-734.

Meade, A.W., Craig, S.B., 2012. Identifying careless responses in survey data. Psychological methods 17, 437.

Meeßen, S.M., Thielsch, M.T., Riehle, D.M., Hertel, G., 2021. Trust is essential: Positive effects of information systems on users' memory require trust in the system. Ergonomics 63, 909-926.

Thielsch, M.T., Röseler, S., Kirsch, J., Lamers, C., Hertel, G., 2021. Managing pandemics - demands, resources, and effective behaviors within crisis management teams. Applied Psychology 70, 150-187.

'This is not a film': Italian mayors rage at virus lockdown dodgers, 2020. The Guardian.

Tingley, D., Yamamoto, T., Hirose, K., Keele, L., Imai, K., 2014. Mediation: R package for causal mediation analysis.

van Mulukom, V., n.d. Low levels of trust affect protective behaviours and conspiracy belief during the COVID-19 pandemic. 